\title{
Frustrated Order by Disorder: the Pyrochlore Antiferromagnet with Bond Disorder
}

\author{
L. Bellier-Castella ${ }^{1}$, M.J.P. Gingras ${ }^{2,3}$, P.C.W. Holdsworth ${ }^{4}$ and R. Moessner ${ }^{5}$ \\ ${ }^{1}$ Departement de Physique des Matériaux, Université Claude Bernard, 69622 Villeurbanne, Cedex, France \\ ${ }^{2}$ Canadian Institute for Advanced Research \\ ${ }^{3}$ Department of Physics, University of Waterloo, 200 University Avenue West, Waterloo, Ontario, Canada N2L $3 G 1$ \\ ${ }^{4}$ Laboratoire de Physique, Ecole Normale Supérieure, 46 Allée d'Italie, F-69364, Lyon, cedex 07, France \\ ${ }^{5}$ Department of Physics, Princeton University, Princeton, NJ 08544, USA
}

(November 16, 2018)

\begin{abstract}
The classical Heisenberg antiferromagnet on the pyrochlore lattice is macroscopically and continuously degenerate and the system remains disordered at all temperatures, even in the presence of weak dilution with nonmagnetic ions. We show that, in stark contrast, weak bond disorder lifts the ground state degeneracy in favour of locally collinear spin configurations. We present a proof that for a single tetrahedron the ground state is perfectly collinear but identify two mechanisms which preclude the establishment of a globally collinear state; one due to frustration and the other due to higher-order effects. We thus obtain a rugged energy landscape, which is necessary to account for the glassy phenomena found in real systems such as the pyrochlore $\mathrm{Y}_{2} \mathrm{Mo}_{2} \mathrm{O}_{7}$ recently reported by Booth et al. [1] to contain a substantial degree of bond disorder.
\end{abstract}

Geometrically frustrated magnets [2], on the kagomé or pyrochlore lattices, are ideal model systems for the study of general concepts pertaining to noncrystalline condensed phases of matter. Behaviour analogous to that of liquid [3], glass [2, 4, 5], and ice [6, 7] phases have already been attributed to experimental pyrochlore systems, while the related Gadolinium Garnet antiferromagnet offers liquid [8] and re-entrant solid behaviour [9]. Equally, theoretical models provide the opportunity to study an array of many body phenomena: the classical Heisenberg antiferromagnet on the pyrochlore lattice retains the behaviour of an idealized liquid right to zero temperature [10], while in the equivalent kagomé 11] system and in the XY antiferromagnet on the pyrochlore lattice 10,12] thermal "order by disorder" effects lead to the apparition of liquid crystal like phases.

Given this rich array of phenomena one would like to present geometrically frustrated magnetism as a field in which one can study charicatures of complex many body systems. However, despite some successes (see for example [2, 9.13]) there remain a number of marked disagreements between experiment and theory which prevent such a desirable coherent picture. For example, experiments on pure $\mathrm{Y}_{2} \mathrm{Mo}_{2} \mathrm{O}_{7}$, which is believed to be well represented by a Heisenberg antiferromagnet on the pyrochlore lattice, show an extremely well defined spin-

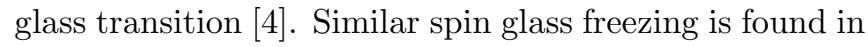
$\mathrm{Tb}_{2} \mathrm{Mo}_{2} \mathrm{O}_{7}$ [5]. This is in sharp contrast to the behaviour of the classical Heisenberg antiferromagnet on the pyrochlore lattice, which shows no sign of glassy behaviour at any temperature [10]. In fact $\mathrm{Y}_{2} \mathrm{Mo}_{2} \mathrm{O}_{7}$ behaves much more like a conventional randomly disordered spin glass or even the disordered compound $\mathrm{CsNiCrF}_{6}$ - which has two kinds of magnetic ion $\left(\mathrm{Cr}^{3+}\right.$ and $\left.\mathrm{Ni}^{2+}\right)$ arranged randomly on a pyrochlore structure - than that anticipated from a collective paramagnet.

Recent x-ray absorption measurements on $\mathrm{Y}_{2} \mathrm{Mo}_{2} \mathrm{O}_{7}$ [1] show that despite the high degree of stoichiometry in this material, there is a small but measurable amount of disorder in the Mo-Mo bond lengths frozen into the system at low temperature. In addition we note that the liquid like diffuse neutron scattering data observed for $\mathrm{CsNiCrF}_{6}$ seem to be best described by locally collinear spin configurations [3]. Thermal selection of a collinear state has been considered as a possibility in the Heisenberg pyrochlore antiferromagnet. However, it has been shown not occur in the disorder-free [10] and site-diluted cases [14].

In this paper we study the effect of weak bond disorder on the classical Heisenberg antiferromagnet on the pyrochlore lattice. We find that bond disorder provides a mechanism for the lifting of the the ground state degeneracy, with the selection of a locally collinear state. This kind of effect is known as configurational order by disorder 15], since the ground states selected by the perturbation typically incorporate long-range order. However, we demonstrate that configurational order by disorder is in turn frustrated on this lattice, since the optimal configuration of individual tetrahedra are not globally compatible, which leads to the collinear order being only of short range. In addition, for finite disorder strengths, the ground states even of individual tetrahedra are no longer necessarily perfectly collinear. A rugged energy landscape arises from the many ways of finding compromises between the competing requirements imposed by the disorder. Our Monte Carlo simulations do indeed find that the development of local collinearity is accompanied by the onset of glassy behaviour.

The Heisenberg Hamiltonian for the pure pyrochlore antiferromagnet is

$$
H_{0}=J \sum_{<i j>} \vec{S}_{i} \cdot \vec{S}_{j}=\frac{J}{2} \sum_{p}\left|\vec{L}_{p}\right|^{2}-N J
$$

where $J>0$ is the coupling constant, $N$ the number of spins and $\vec{L}_{p}$ is the total spin on the $p^{t h}$ tetrahedron. The 
sum on $\langle i j\rangle$ runs over all nearest neighbor bonds. The ground state condition $\vec{L}_{p} \equiv 0$ leaves one degree of freedom per tetrahedron unconstrained, thus yielding a continuously degenerate ground state of extensive dimension $N / 2$ and localised zero-energy degrees of freedom which fluctuate all the way down to $T=0$ [10.

Thermal order by disorder, in which ordered states are selected at small but nonzero temperature due to the large entropy of the soft fluctuations around them, has already been discussed for this model [10,16]. The candidate states for selection are the discrete subset of collinear ground states, which have the broken uniaxial symmetry of a nematic and are exponentially numerous in $N$. However, localising the system in phase space near points of this discrete set of collinear states is prohibitively costly in entropy, even taking into account the soft fluctuations around the collinear states: thermal order by disorder, unusually for frustrated magnets, is absent here.

We add a small disordered term, $H_{1}$, to $H_{0}$ :

$$
H_{1}=J \sum_{<i, j>} \delta_{i, j} \vec{S}_{i} \cdot \vec{S}_{j} ; \quad-\delta_{0}<\delta_{i, j}<\delta_{0},
$$

with $\delta_{i, j}$ taken from a continous and rectangular distribution. We show, in the inset of Fig. 1, the energy per spin at low temperatures $(\mathrm{T} / \mathrm{J}=0.0006)$ for different disorder strengths. As $\delta_{0}$ increases, the energy per spin decreases linearly, illustrating that the disorder relieves the frustration, allowing lower energy states for specific configurations. One can see that these should be collinear by recognizing that the energy on a given tetrahedron is minimized if the spins on the weakest bonds (i.e those with $\delta_{i, j}$ most negative) are ferromagnetically aligned, while those on the strongest bonds are antiferromagnetically aligned. The energy of a single tetrahedron can therefore be reduced compared to a non-collinear state by judicious selection of which spins are up and which are down. In the appendix we give a proof that the lowest energy state is indeed a collinear state for a single tetrahedron in the limit of weak disorder.

As a test for the formation of collinear order we have calculated the probability density function $P(w)$ for the scalar product between neighbouring spin vectors, $w_{i, j}=$ $\vec{S}_{i} \cdot \vec{S}_{j}$, by Monte Carlo simulation. For collinear states with 4 antiferromagnetic and 2 ferromagnetic bonds per tetrahedron, $P(w)$ is bimodal with delta functions of strength $2 / 3$ and $1 / 3$ at $w=-1$ and $w=1$ respectively. In Fig. 1 we compare $P(w)$ for a system of 1024 spins in a cubic cell with periodic boundaries for a system without disorder and for a system with $\delta_{0} / J=0.1$. In both cases, the data were collected over $10^{6}$ Monte Carlo steps per spin (MCS) at $T / J=0.001$ and the initial configuration generated by cooling in stages of $10^{5}$ MCS. In the pure case on reducing the temperature a peak develops at $w=-1$ indicating the presence of weak local antiparallel correlations. In the presence of bond disorder the low temperature feature at $w=-1$ is much more pronounced. In addition a clear peak in probility is present also at $w=1$, as expected from the configurational order by disorder. Nonetheless, collinear ordering clearly remains incomplete.

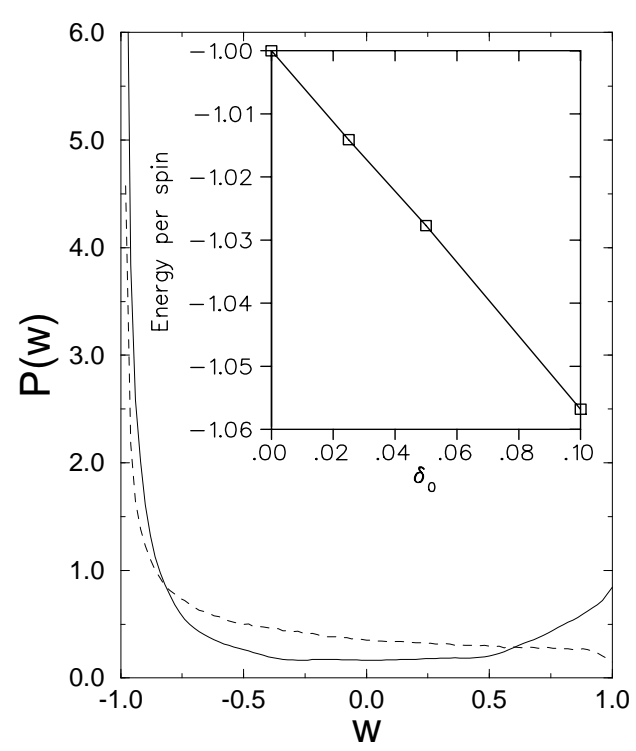

FIG. 1. Probability density function $P(w)$ for 1024 spins. The full line is for $\delta_{0}=0.1$ and the dotted line for $\delta_{0}=0$. The inset shows the energy per spin with bond energy taken from the distribution (Eq. 2). Values are from $N=1024$ spin systems averaged over 10 disorder configurations.

To see why this is the case, note that global collinear order by disorder is frustrated by the presence of closed loops of bonds on the lattice. If the single-tetrahedron ground states imply an odd number of antiparallel bonds around any closed loop, a global ground state obviously cannot be constructed consistently out of those for single tetrahedra.

Collinear order could be retained at the cost of having higher-energy configurations on some of the tetrahedra, but this strategy is not promising due to the large number of closed loops of bonds. The alternative is to introduce canting, and this is indeed what happens, as borne out by our simulations results. The large number of possible compromises imposed by the loops leads to a large number of states with similar energy but separated by barriers - a rugged energy landscape.

To leading order in $\delta_{0}$ the local energy minima lie on the original ground state manifold with $\vec{L}_{p}=0$, while to higher order they move off this surface towards the minima of $H_{1}$. For collinear states the spectrum of excitations of $H_{0}$ perpendicular to this surface contains soft modes, and therefore canting away from collinearity due to higher order effects can be considerable, even for modest values of $\delta_{0}$. The higher order effects therefore provide a mechanism for the destruction of collinearity, even for a single tetrahedron and even for modest values of $\delta_{0}$.

We have tested for both spin glass behaviour and global collinearity, since the ground state manifold is now disconnected due to the formation of energy barriers which could induce glassy behaviour and loss of ergodicity.

The nematic order parameter $Q$, is the largest eigenvalue of the traceless matrix 18]

$$
Q_{\alpha, \beta}=\frac{3}{2 N} \sum_{i}<\left(\vec{S}_{i}\right)_{\alpha}\left(\vec{S}_{i}\right)_{\beta}>-\frac{1}{2} \delta_{\alpha \beta},
$$

where $\alpha=x, y, z$ and $\delta_{\alpha \beta}$ is the Kronecker delta. The 
Edwards-Anderson spin glass order parameter [19] is

$$
q_{E A}=\frac{1}{N} \sum_{i}<\vec{S}_{i}>^{2}
$$

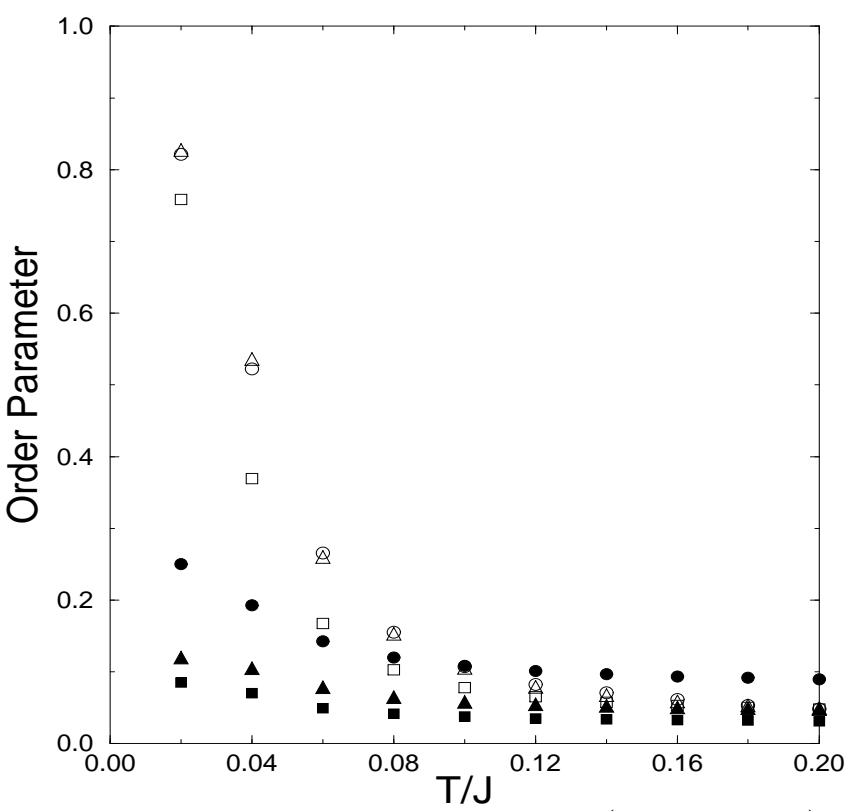

FIG. 2. Nematic order parameter $Q$ (filled symbols)and spin glass order parameters $q^{E A}$ (open symbols) against temperature, calculated for 128 spins (circles), 432 spins (triangles) and 1024 spins (squares).

In Fig. 2 we show $Q$ and $q_{E A}$ against temperature for $\delta_{0}=0.1$ for $N=128,432$ and 1024 spins. The data is averaged over $10-30$ configurations of disorder, with at each temperature $10^{5}$ MCS for equilibration and $10^{5}$ for production. The data clearly show the onset of glassy behaviour at a temperature of the order of $T / J=\delta_{0} / J=0.1$. There is a marked decrease of $q_{E A}(T)$ for $T<0.08$ from $N=432$ to $N=1024$, suggesting that the freezing occurs over microscopic clusters only. Moreover, preliminary results for the susceptibility suggest, at least for small systems, that there is no bifurcation between field-cooled and zero field cooled measurements. However, the time scales involved do increase by orders of magnitude in the presence of bond disorder: the relaxation time at the lowest temperature studied, $T / J=0.02$ is in excess of $10^{6} \mathrm{MCS}$, while for the pure system it is of order $10^{3}$ MCS.

The nematic order parameter scales to zero at low temperature roughly as $Q(T=0) \sim 4 / \sqrt{N}$ confirming that the collinearity is only a local phenomena, with correlations over about 4 tetrahedra.

We conclude that, although the ground state for a single tetrahedron is perfectly ordered, the state attained for the larger system is only partially ordered and is dynamically frozen on the time scales we have considered. This may not only be because the true ground state is not perfectly collinear, as discussed above, but also because a loss of ergodicity in the simulations can mean that the system gets stuck in a metastable state.

Our results therefore provide a plausible mechanism for the local collinearity proposed to fit the neutron scattering data of Harris et al on $\mathrm{CsNiCrF}_{6}$ [3]. Bond disorder alone with isotropic Heisenberg spins is probably not sufficient to explain the glassy behaviour observed in $\mathrm{Y}_{2} \mathrm{Mo}_{2} \mathrm{O}_{7}$. However it is sufficient to remove the anomalous liquid like behaviour of the classical Heisenberg antiferromagnet and ensure dynamical freezing at a well defined temperature, for any finite system. One might naively expect that, due to absence of long-range nematic order, the present system renormalizes to an effective randomly frustrated isotropic Heisenberg spin glass, at length scales beyond the short-range correlated collinear regions. It is generally believed that the isotropic three dimensional Heisenberg spin glass has a zero temperature spin-glass freezing transition [20], with no field-cool/zero field-cool split in the magnetization as is also indicated by our simulations. Within this picture, the observed spin glass transition in $\mathrm{Y}_{2} \mathrm{Mo}_{2} \mathrm{O}_{7}$ 相 must come from anisotropic perturbations 21. Site disorder has also been considered previously [14], but it does not lead to glassy behaviour: substitution of a magnetic ion does not destroy the continuous ground state manifold but only reduces its dimension. Bond disorder, on the other hand, should lead to energy barriers of a height set by the disorder strength, which is just what we find in Fig. 2. More work is clearly required here. In particular, further simulations are needed to investigate the nature of the glass transition in more detail. It would also be of interest to re-examine neutron data with a view to fitting $S(q, \omega)$ using local collinear spin configurations.

Finally we note that the longest standing theoretical challenge in this field is motivated by the most studied experimental system $\mathrm{SrCr}_{x} \mathrm{Ga}_{12-x} \mathrm{O}_{19}$ (SCGO) which consists of bi-layers of two kagomé lattices of magnetic ions which are coupled, forming a slice of pyrochlore lattice. There is experimental evidence that, at low temperature the spins form into a glassy co-planar phase [2,22,23] but no theoretical explanation for this result exists. The experimental compound is non-stoichiometric and so substitutional disorder is inevitable. The thermal order by disorder seen in the classical kagomé [11] antiferromagnet has been shown to be extremely sensitive to disorder [24], with $2 \%$ dilution being sufficient to destroy coplanarity, and so cannot be responsible for the observed coplanarity. Further to this, in presence of the $12 k-2 a-12 k$ kagomé-triangular-kagomé coupling, the coplanar thermal order-by-disorder found for a single kagomé layer is destroyed, with or without site or bond disorder. It is therefore tempting to speculate that a variant of the mechanism presented here could lead to configurational coplanar order by disorder in parallel with glassy behaviour, in agreement with experiment at long last. However, we have not, as yet, managed to isolate such a variant. 
This reserach has been funded by NSERC of Canada and the Pole Scientifique de Modélisation Numérique (PSMN) of the Ecole Normale Superieure de Lyon. M.G. and P.H. are grateful to the Association of Universities and Colleges of Canada for a travel grant from the Going Global STEP program. M.G. acknowledges the Research Corporation for a Research Innovation Award and a Cottrell Scholar Award, and the Province of Ontario for a Premier Research Excellence Award.

\section{APPENDIX A:}

We consider the Hamiltonian $H=H_{0}+H_{1}$ for a single tetrahedron, and treat $H_{1}$ in first order of perturbation theory. The ground states of $H_{0}$ have total spin $\vec{L}=0$. They can be parametrized as $\vec{S}_{1}=(0,0,1) ; \vec{S}_{2}=$ $(0, \sin \alpha, \cos \alpha) ; \vec{S}_{3}=(\sin \beta \sin \phi, \sin \beta \cos \phi, \cos \beta) ; \vec{S}_{4}=$ $-\vec{S}_{1}-\vec{S}_{2}-\vec{S}_{3}$. The normalisation of $\vec{S}_{4}$ imposes the restriction $\cos \alpha+\cos \beta \leq 0$, as well as a condition on $\phi[17$. Therefore,

$$
\begin{aligned}
& \vec{S}_{1} \cdot \vec{S}_{2}=\cos \alpha \\
& \vec{S}_{1} \cdot \vec{S}_{3}=\cos \beta \\
& \vec{S}_{1} \cdot \vec{S}_{4}=-1-\cos \alpha-\cos \beta .
\end{aligned}
$$

In a ground state of $H_{0}$, the sum of any pair of spins is opposite to the sum of the other pair of spins, and hence

$$
\vec{S}_{3} \cdot \vec{S}_{4}=\vec{S}_{1} \cdot \vec{S}_{2} \quad, \quad \vec{S}_{2} \cdot \vec{S}_{4}=\vec{S}_{1} \cdot \vec{S}_{3} \quad, \quad \vec{S}_{2} \cdot \vec{S}_{3}=\vec{S}_{1} \cdot \vec{S}_{4}
$$

The dot products between two spins can thus be expressed using $\cos \alpha$ and $\cos \beta$ only, this means that, restricted to the ground states of $H_{0}, H_{1}$ must have the following form:

$$
H_{1} / J=A \cos \alpha+B \cos \beta+C,
$$

where $A, B$ and $C$ depend on the $\delta: A=\delta_{1,2}+\delta_{3,4}-\delta_{1,4}-$ $\delta_{2,3}, B=\delta_{1,3}+\delta_{2,4}-\delta_{1,4}-\delta_{2,3}$, and $C=-\delta_{1,4}-\delta_{2,3}$.

We now have to distinguish the following cases: (I) $A \neq B$. Without loss of generality, let $A>B$. (a) For $A>0, B \neq 0$, the global minimum of $H_{1}$ is given by $\cos \alpha=-1$ and $\cos \beta=-\operatorname{sign}(B)$. (b) If $A>0, B=$ 0 , then $\cos \beta$ is undetermined. (c) For $A<0, H_{1}$ is minimized by $\cos \alpha=-1, \cos \beta=1$. (II) $A=B$ (a) $A>0$ : $H_{1}$ is minimized by $\cos \alpha=\cos \beta=-1$. (b) $A<0$ : $H_{1}$ is minimized by $\cos \alpha+\cos \beta=0$. (c) $A=0$ : $H_{1}$ is independent of $\alpha, \beta$.

From this, we see that two things can happen. Either all spins are collinear, in the cases Ia,Ic,IIa, or the degeneracy is not fully lifted, in the special (finetuned) cases Ib,IIb,IIc. For the latter, a collinear state still exists which minimizes the energy. In other words, a noncollinear state is never selected by the disorder as unique ground state, and the generic case $A \neq B, A B \neq 0$ has a unique ground state which is collinear.
[1] C.H. Booth, J.S. Gardner, G.H. Kwei, R.H. Heffner, F. Bridges and M.A. Subramanian, to appear in Phys. Rev. B.

[2] For reviews, see: A. P. Ramirez, Annu. Rev. Mater. Sci. 24, 453, (1994); P. Schiffer and A. P. Ramirez, Comments Cond. Mat. Phys. 18, 21 (1996).

[3] M.J. Harris, M.P. Zinkin, Z.Tun, B.M. Wanklyn and I.P. Swainson, Phys. Rev. Lett. 73, 189 (1994); M.P. Zinkin, M.J. Harris and T. Zeiske, Phys. Rev. B 56, 11786 (1997).

[4] S.R. Dunsiger et al., Phys. Rev. B 54, 9019 (1996); M.J.P. Gingras et al., Phys. Rev. Lett. 78, 947 (1997); J.S. Gardner et al., Phys. Rev. Lett. 83, 211 (1999).

[5] B. D. Gaulin, J. N. Reimers, T. E. Mason, J. E. Greedan and Z. Tun, Phys. Rev. Lett. 69, 3244 (1992).

[6] M.J. Harris, S.T. Bramwell, D.F. McMorrow, T. Zeiske and K.W. Godfrey, Phys. Rev. Lett. 79, 2554 (1997).

[7] A. Ramirez, A. Ayashi, R. Cava, R. Siddarthan and B. Shastry, Nature 399, 333 (1999).

[8] O.A. Petrenko, C. Ritter, M. Yethiraj and D. McK Paul, Phys. Rev. Lett. 80, 4570 (1998).

[9] P. Shiffer, A.P. Ramirez, D.A. Huse and A.J. Valentino, Phys. Rev. Lett. 73, 2500 (1994).

[10] R. Moessner and J.T. Chalker, Phys. Rev. Lett. 80, 2929 (1998); Phys. Rev. B 58, 12049 (1998).

[11] J.T. Chalker, P.C.W. Holdsworth and E.F. Shender, Phys. Rev. Lett. 68, 855 (1992); J.N. Reimers and A.J. Berlinsky, Phys. Rev. B 48, 9539 (1993); I. Ritchey, P. Chandra and P. Coleman, Phys. Rev. B 47, 15342 (1993); A.B. Harris, C. Kallin and A.J. Berlinksy, Phys. Rev. B 45, 2899 (1992).

[12] S.T. Bramwell, M.J.P. Gingras and J.N. Reimers, J. Appl. Phys. 75, 5523 (1994).

[13] B. C. den Hertog and M. J. P. Gingras, Phys. Rev. Lett. 84, 3430 (2000).

[14] R. Moessner and A.J. Berlinsky, Phys. Rev. Lett. 83, 3293 (1999).

[15] J. Villain, R. Bidaux, J.P. Carton and R.J. Conté, J. Phys. (Paris) 41, 1263 (1980).

[16] J.N. Reimers, Phys. Rev. B 45, 7287 (1992).

[17] J. N. Reimers, A. J. Berlinsky and A.-C. Shi, Phys. Rev. B 43, 865 (1991).

[18] M. Plischke and B. Bergersen, "Equilibrium Statistical Physics", World Scientific, 1994.

[19] K.H. Fischer and J.A. Hertz, "Spin Glasses", Cambridge University Press, Cambridge (1991).

[20] J.A. Olive, A.P. Young and D. Sherrington, Phys. Rev. B 34, 6341 (1986).

[21] M.J.P. Gingras, Phys. Rev. Lett. 71, 1637 (1993).

[22] A. P. Ramirez, G.P. Espinosa and A.S. Cooper, Phys. Rev. Lett. 64, 2070 (1990).

[23] P. Schiffer, A. P. Ramirez, K. N. Franklin and S-W. Cheong, Phys. Rev. Lett. 77, 2085 (1996).

[24] E.F. Shender, V.B. Cherepenov, P.C.W. Holdsworth and A.J. Berlinsky, Phys. Rev. Lett. 70, 3812 (1993). 